\title{
Genes encoding critical transcriptional activators for murine neural tube development and human spina bifida: a case-control study
}

\author{
Wei Lu', Adrian R Guzman', Wei Yang ${ }^{2}$, Claudia J Chapa ${ }^{5}$, Gary M Shaw², Robert M Greene ${ }^{3}$, M Michele Pisano $^{3}$, \\ Edward J Lammer ${ }^{4}$, Richard H Finnell ${ }^{5}$, Huiping Zhu ${ }^{5^{*}}$
}

\begin{abstract}
Background: Spina bifida is a malformation of the neural tube and is the most common of neural tube defects (NTDs). The etiology of spina bifida is largely unknown, although it is thought to be multi-factorial, involving multiple interacting genes and environmental factors. Mutations in transcriptional co-activator genes-Cited2, p300, Cbp, Tfap2 $\alpha$, Carm 1 and Cart1 result in NTDs in murine models, thus prompt us to investigate whether homologues of these genes are associated with NTDs in humans.
\end{abstract}

Methods: Data and biological samples from 297 spina bifida cases and 300 controls were derived from a population-based case-control study conducted in California. 37 SNPs within CITED2, EP300, CREBBP, TFAP2A, CARM1 and $A L X 1$ were genotyped using an ABI SNPlex assay. Odds ratios and $95 \%$ confidence intervals were calculated for alleles, genotypes and haplotypes to evaluate the risk for spina bifida.

Results: Several SNPs showed increased or decreased risk, including CITED2 rs1131431 (OR $=5.32,1.04 \sim 27.30$ ), EP300 rs4820428 ( $\mathrm{OR}=1.30,1.01 \sim 1.67)$, EP300 rs4820429 ( $\mathrm{OR}=0.50,0.26 \sim 0.50$, in whites, $\mathrm{OR}=0.7,0.49 \sim 0.99$ in all subjects), EP300 rs17002284 (OR = 0.43, 0.22 0.84), TFAP2A rs3798691 (OR = 1.78, 1.13 2.87 in Hispanics), CREBBP rs129986 (OR = 0.27, 0.11 0.69), CARM1 rs17616105 (OR = 0.41, 0.22 0.72 in whites). In addition, one haplotype block in EP300 and one in TFAP2A appeared to be associated with increased risk.

Conclusions: Modest associations were observed in CITED2, EP300, CREBBP, TFAP2A and CARM1 but not ALX1. However, these modest associations were not statistically significant after correction for multiple comparisons. Searching for potential functional variants and rare causal mutations is warranted in these genes.

\section{Background}

Studies using genetically modified mouse models have contributed a great deal to our understanding of molecular mechanisms that govern critical embryonic development processes, such as neural tube closure. Among the over 200 existing mouse models for neural tube defects (NTDs), several harbor mutations in genes encoding transcriptional activators or co-activators, such as Cited2, Crebbp, p300, Tfap 2 $\alpha$, Carm1 and Cart1 [1-9].

CREB binding protein (CREBBP; CBP) was originally identified as a protein that binds to the phosphorylated form of the CREB transcription factor and increases the

\footnotetext{
* Correspondence: hzhu@austin.utexas.edu

${ }^{5}$ Dell Pediatric Research Institute, UT Austin, Austin, TX, USA

Full list of author information is available at the end of the article
}

expression of genes containing CRE elements. It is closely related to the adenovirus E1A-associated protein p300. CBP and p300 are functionally redundant histone deacetylases essential for proliferation and embryonic development $[5,8,10]$. Both Crebbp and $p 300$ genes are expressed at high level in the neural folds at embryonic day E8.5 in mice [3]. Homozygous deletions of either the Crebbp or $p 300$ genes in mice results in lethality on E9 11, with subsequent defects in neurulation, cell proliferation and cardiac development $[5,8]$.

The methylation of the Crebbp/p300 complex is catalyzed by co-activator-associated arginine methyltransferase (CARM1). This process disables the recruitment of cAMP response element binding protein (CREB). Thus, CARM1 functions as a co-repressor in the cAMP signaling 
pathway, via its methyltransferase activity [11]. In addition to its enzymatic function, CARM1 is also required for NFkappaB-dependent gene expression as a transcriptional cofactor [12]. Carm1 gene expression was observed in the fore- and hindbrain, neural folds, somites and posterior lateral plates of E8.25 mouse embryos, and later on in development (E8.75) expression became prominent in the neural tube and somites [13].

CITED2 is a nuclear co-activator that binds to CBP/ p300. Disruption of the Cited2 gene in mice is embryonic lethal, with the mice dying secondary to significant defects in the development of the neural tube and the heart $[2,6,14,15]$. The interaction of CBP and p300 with Cited 2 is required for activation of important transcriptional factors such as AP- $2 \alpha[1,16]$. Mice carrying homozygous disruptions of the gene coding for the protein AP-2 $\alpha($ Tfap $2 \alpha)$ die at term as a result of severe developmental abnormalities. The earliest defect observed was a complete failure of neural tube closure in the cranial region, and it has been suggested in a chimera model study that there is an independent requirement for AP-2 $\alpha$ in the formation of the neural tube [17-19].

CART1 is a paired-class homeobox-containing transcription factor regulated by CBP/p300 acetylation of a highly conserved lysine residue that increases the interaction between $\mathrm{CBP} / \mathrm{p} 300$ and CART1, ultimately enhancing its transcriptional activation [20]. The Cart1 gene is expressed in chondrocytes [21,22], and appears to be required for embryonic forebrain mesenchymal survival. The absence of CART1 disrupts cranial neural tube morphogenesis by blocking the initiation of closure in the midbrain region. Homozygous Cart 1 mutant mice are born alive with neural tube closure defects including acrania and meroanencephaly, but die soon after birth [9]. The Cart1 knockout mouse is among several NTD mouse models that can be rescued by maternal supplementation with folic acid [23]. The gene encoding human CART1 is $A L X 1$ (ALX homobox 1). In summary, CBP, p300, CARM1, TFAP2 $\alpha$, CITED2 and CART1 are transcriptional activator/co-activators that contributes to the ontogeny of the neural tube in mammalian systems. Whether gene homologues are associated with human NTDs remains unknown. Three single nucleotide polymorphisms (SNP) in the un-translated region of CITED2 gene were studied in a group of patients with spina bifida and controls, modest associations were observed but results were imprecise owing to small sample size [14]. Mutations in the human $C B P$ gene have been identified as being responsible for the Rubinstein-Taybi syndrome (RTS), which has a defined craniofacial phenotype [4]. The purpose of this study is to evaluate the potential association between variations among human CITED2, CREBBP, EP300, TFAP2A, $C A R M 1$ and $A L X 1$ genes and the risk for spina bifida.

\section{Methods}

\section{Study Population}

Data and biological samples were obtained from a casecontrol study conducted by the California Birth Defects Monitoring Program (CBDMP). The CBDMP is an active, population-based surveillance system for collecting information on infants and fetuses with structural congenital malformations, which has been described elsewhere [24]. Included were 297 singleton infants with spina bifida (cases) and 300 non-malformed infants (controls). Both cases and controls were randomly selected from the samples with bloodspots available. Cases were selected from California Birth Defect Registry 1990-1999; nonmalformed controls were selected from Vital Statistics matching the birth years with the selected cases. Case and control infants were linked to their newborn screening bloodspots. All samples were obtained with approval from the State of California Health and Welfare Agency Committee for the Protection of Human Subjects.

\section{Candidate genes and SNPs}

The 6 candidate genes and SNPs genotyped are listed in Table 1. Tagging SNPs were selected for each candidate gene using the SNPbrowser ${ }^{\mathrm{rm}}$ Software (ABI, Foster City, CA) based on data from the four HapMap populations (CEU, YRI, JPT and CHB) [25]. All validated tagging SNPs with pairwise $r^{2}>=0.8$ with a minor allele frequency (MAF) no less than 5\% were included for genotyping. Also included for study were all non-synonymous coding SNPs in these genes. Based on these criteria, 37 SNPs were genotyped.

\section{Genotyping Approach}

DNA was extracted from blood spots using the Gentra Puregene DNA Extraction Kit (Qiagen, Valencia, CA) following the standard protocol. Prior to genotyping, genomic DNA was amplified using a commercially available multiple displacement amplification (MDA) kit, GenomiPhi DNA Amplification kit (GE Healthcare, Piscataway, NJ). The whole genome amplification (WGA) product was then quantified using RNase $\mathrm{P}$ assay (AppliedBiosystems, Foster City, CA). 200 ng of WGA product was used for the SNPlex assay containing the 37 SNPs. All genotyping assays were performed blinded to subjects' case or control status.

The SNPs selected for analyses were submitted to Applied Biosystems to create a unique SNPlex assay. The SNPlex $^{\mathrm{Tm}}$ genotyping system (Applied Biosystems, Foster City, CA) is based on a universal multiplexed oligonucleotide ligation assay (OLA)/PCR and drag chute mobility modifier technology. The resulting genotype data were analyzed using GeneMapper ${ }^{\mathrm{TM}}$ v4.0 (Applied Biosystems, Foster City, CA). 
Table 1 Characteristics of all genotyped SNPs

\begin{tabular}{|c|c|c|c|c|c|c|c|c|c|c|c|}
\hline $\begin{array}{l}\text { Gene } \\
\text { (Alias) }\end{array}$ & Marker & Chromosome & $\begin{array}{c}\text { Chromosome } \\
\text { position }\end{array}$ & $\begin{array}{l}\text { RefSNP } \\
\text { alleles }\end{array}$ & $\begin{array}{c}\text { Call } \\
\text { Rate } \\
(\%) \\
\end{array}$ & $\begin{array}{c}\text { MAF* }^{*} \\
\text { (All } \\
\text { subjects) }\end{array}$ & $\begin{array}{c}\text { HWE P } \\
\text { (controls) }\end{array}$ & $\begin{array}{c}\text { MAF } \\
\text { (White) }\end{array}$ & $\begin{array}{c}\text { HWE }^{* *} \mathrm{P} \\
\text { (White } \\
\text { controls) }\end{array}$ & $\begin{array}{c}\text { MAF } \\
\text { (Hispanic) }\end{array}$ & $\begin{array}{c}\text { HWE P } \\
\text { (Hispanic } \\
\text { controls) }\end{array}$ \\
\hline \multirow{3}{*}{$\begin{array}{l}\text { ALX1 } \\
\text { (CART1) }\end{array}$} & rs11608773 & 12 & 84229006 & $\mathrm{~A} / \mathrm{G}$ & 100.0 & 0.229 & 0.435 & 0.274 & 0.478 & 0.217 & 0.391 \\
\hline & rs4761130 & 12 & 84226396 & $C / T$ & 99.8 & 0.421 & 0.286 & 0.358 & 0.154 & 0.401 & 0.465 \\
\hline & rs7295242 & 12 & 84222948 & $\mathrm{~A} / \mathrm{G}$ & 100.0 & 0.161 & 0.781 & 0.074 & 0.557 & 0.159 & 0.358 \\
\hline \multirow[t]{6}{*}{ CARM1 } & rs11667234 & 19 & 10880581 & $\mathrm{~A} / \mathrm{C}$ & 99.8 & 0.021 & 0.010 & 0.039 & 0.071 & 0.016 & 0.934 \\
\hline & rs11670365 & 19 & 10865833 & $\mathrm{C} / \mathrm{T}$ & 100.0 & 0.056 & 0.918 & 0.084 & 0.754 & 0.045 & 0.702 \\
\hline & rs1529711 & 19 & 10884434 & $\mathrm{~A} / \mathrm{G}$ & 99.7 & 0.097 & 0.492 & 0.161 & 0.932 & 0.081 & 0.519 \\
\hline & rs1541596 & 19 & 10848013 & $\mathrm{~A} / \mathrm{G}$ & 99.5 & 0.475 & 0.326 & 0.497 & 0.969 & 0.408 & 0.957 \\
\hline & rs17616105 & 19 & 10883159 & $\mathrm{C} / \mathrm{T}$ & 100.0 & 0.189 & 0.732 & 0.235 & 0.365 & 0.152 & 0.072 \\
\hline & rs7254708 & 19 & 10863579 & $\mathrm{~A} / \mathrm{G}$ & 100.0 & 0.071 & 0.043 & 0.123 & 0.116 & 0.059 & 0.441 \\
\hline \multirow[t]{2}{*}{ CITED2 } & rs1131431 & 6 & 139735527 & $\mathrm{~A} / \mathrm{G}$ & 100.0 & 0.153 & 0.273 & 0.200 & 0.624 & 0.121 & 0.948 \\
\hline & rs7775752 & 6 & 139737486 & $A / G$ & 45.1 & 0.007 & 0.957 & 0.009 & 0.913 & 0.006 & 1.000 \\
\hline \multirow{9}{*}{$\begin{array}{l}\text { CREBBP } \\
(C B P)\end{array}$} & rs11076783 & 16 & 3722706 & $C / T$ & 100.0 & 0.154 & 0.638 & 0.116 & 0.142 & 0.139 & 0.802 \\
\hline & rs11076785 & 16 & 3730187 & $\mathrm{G} / \mathrm{T}$ & 46.6 & 0.457 & 0.638 & 0.401 & 0.185 & 0.457 & 0.463 \\
\hline & rs11076786 & 16 & 3751597 & $\mathrm{C} / \mathrm{T}$ & 100.0 & 0.075 & 0.008 & 0.077 & 0.441 & 0.046 & 0.637 \\
\hline & rs129986 & 16 & 3744242 & $C / T$ & 100.0 & 0.023 & 0.530 & 0.032 & 0.590 & 0.020 & 0.735 \\
\hline & rs130003 & 16 & 3768173 & $\mathrm{~A} / \mathrm{G}$ & 99.8 & 0.015 & 0.792 & 0.032 & 0.711 & 0.010 & 0.967 \\
\hline & rs130008 & 16 & 3721953 & $\mathrm{C} / \mathrm{G}$ & 99.8 & 0.011 & 0.838 & 0.032 & 0.752 & 0.001 & 1.000 \\
\hline & rs17136507 & 16 & 3740546 & $\mathrm{C} / \mathrm{T}$ & 100.0 & 0.178 & 0.536 & 0.110 & 0.229 & 0.227 & 0.449 \\
\hline & rs886528 & 16 & 3751557 & $C / T$ & 99.7 & 0.383 & 0.590 & 0.390 & 0.986 & 0.368 & 0.839 \\
\hline & rs9392 & 16 & 3715170 & $\mathrm{~A} / \mathrm{G}$ & 99.8 & 0.189 & 0.566 & 0.223 & 0.625 & 0.138 & 0.700 \\
\hline \multirow{8}{*}{$\begin{array}{l}\text { EP300 } \\
\text { (P300) }\end{array}$} & rs17002284 & 22 & 39826792 & $\mathrm{~A} / \mathrm{G}$ & 100.0 & 0.265 & 0.220 & 0.313 & 0.745 & 0.217 & 0.522 \\
\hline & rs17433014 & 22 & 39829768 & $\mathrm{~A} / \mathrm{G}$ & 99.8 & 0.018 & 0.769 & 0.029 & 0.793 & 0.019 & 0.834 \\
\hline & rs2230111 & 22 & 39851949 & $\mathrm{~A} / \mathrm{G}$ & 99.8 & 0.002 & 0.977 & 0.006 & 0.959 & 1.000 & 1.000 \\
\hline & rs4820428 & 22 & 39867535 & $\mathrm{~A} / \mathrm{G}$ & 100.0 & 0.446 & 0.006 & 0.329 & 0.822 & 0.419 & 0.667 \\
\hline & rs4820429 & 22 & 39868057 & $C / G$ & 100.0 & 0.159 & 0.382 & 0.258 & 0.040 & 0.124 & 0.471 \\
\hline & rs4822006 & 22 & 39849308 & $C / T$ & 100.0 & 0.247 & 0.844 & 0.355 & 0.587 & 0.185 & 0.740 \\
\hline & rs5758246 & 22 & 39871792 & $\mathrm{~A} / \mathrm{G}$ & 100.0 & 0.030 & 0.635 & 1.000 & 1.000 & 0.012 & 0.934 \\
\hline & rs9611502 & 22 & 39861182 & $C / T$ & 100.0 & 0.014 & 0.838 & 0.019 & 0.918 & 0.013 & 0.867 \\
\hline \multirow{9}{*}{$\begin{array}{l}\text { TFAP2A } \\
\text { (AP2) }\end{array}$} & rs10456013 & 6 & 10506548 & $\mathrm{G} / \mathrm{T}$ & 100.0 & 0.032 & 0.592 & 0.048 & 0.670 & 0.026 & 0.735 \\
\hline & rs1621700 & 6 & 10528084 & $\mathrm{~A} / \mathrm{G}$ & 99.7 & 0.288 & 0.671 & 0.252 & 0.081 & 0.357 & 0.773 \\
\hline & rs303050 & 6 & 10511169 & $\mathrm{C} / \mathrm{T}$ & 100.0 & 0.257 & 0.061 & 0.165 & 0.259 & 0.262 & 0.267 \\
\hline & rs303055 & 6 & 10527462 & $\mathrm{C} / \mathrm{T}$ & 100.0 & 0.353 & 0.150 & 0.435 & 0.569 & 0.335 & 0.025 \\
\hline & rs303056 & 6 & 10528467 & $\mathrm{C} / \mathrm{G}$ & 100.0 & 0.159 & 0.229 & 0.094 & 0.477 & 0.126 & 1.000 \\
\hline & rs3798691 & 6 & 10519381 & $\mathrm{C} / \mathrm{G}$ & 100.0 & 0.112 & 0.021 & 0.042 & 0.011 & 0.134 & 0.111 \\
\hline & rs3798694 & 6 & 10516743 & $\mathrm{~A} / \mathrm{G}$ & 100.0 & 0.062 & 0.122 & 0.087 & 0.225 & 0.059 & 0.293 \\
\hline & rs533558 & 6 & 10503558 & $\mathrm{~A} / \mathrm{G}$ & 100.0 & 0.441 & 0.848 & 0.361 & 0.955 & 0.481 & 0.858 \\
\hline & rs537112 & 6 & 10503192 & $\mathrm{~A} / \mathrm{G}$ & 100.0 & 0.180 & 0.571 & 0.103 & 0.754 & 0.195 & 0.255 \\
\hline
\end{tabular}

*MAF: minor allele frequency; **HWE: Hardy-Weinberg Disequilibrium.

\section{Analytic procedures}

Analyses were performed for the overall study group as well as for specific ethnic/race groups. Hispanic infants were further subdivided into those whose mothers were born in the United States, or native-born (Hisp-NB) and those whose mothers were not, or foreign-born (Hisp-FB), as it has been shown that these two groups are different with respect to ethnic composition and social economic 
status [26,27]. Departure from Hardy-Weinberg Equilibrium (HWE) was assessed by Chi-square analysis in each subgroup using data from controls only. Odds ratios (OR) for SNP genotypes were calculated using logistic regression analyses. Since the mode of inheritance is unknown, potential associations with spina bifida risk were evaluated assuming dominant, recessive and additive models for each SNP. Chi-square tests were used to assess the significance of dominant and recessive models. CochranArmitage trend tests were used to assess the significance of additive models. If numbers were less than 5, Fisher's exact test and Exact Armitage trend test were applied. Analyses were performed using SVS v7.1.1 (GoldenHelix, Bozeman, MT) software. The false discovery rate (FDR) [28] was used to account for multiple testing (data not shown). Haplotype blocks were constructed using HaploView 4.2 http://www.broadinstitute.org/haploview/ haploview in each race/ethnicity group with pair-wise $D^{\prime} \geq 0.85$. Haplotype frequencies were estimated based on the Maximum Expectation (EM) algorithm. Association of each haplotype was evaluated by calculating ORs and 95\% confidence intervals against all other haplotypes. Estimated risks were considered statistically precise if $95 \%$ confidential interval of OR does not include 1.00.

\section{Results and Discussion}

Demographic characteristics of the cases and controls are listed in Table 2. Cases and controls were similar with respect to maternal age, parity, and plurality but expectedly differed with respect to race/ethnicity and maternal educational attainment. That is, the data revealed greater frequencies of Hispanics (primarily foreign born) and women with low educational level (less than high school) in the spina bifida case group.

Genotyping of 37 SNPs was performed using a custommade Applied Biosystems SNPlex panel. Due to low call rates, two SNPs, rs7775752 (45.1\%) and rs11076785 (46.6\%), were excluded from analysis. Departure from HWE in the controls $(p<0.01)$ was observed in rs11076786 and rs4820428 when analyzing the whole dataset. This deviation was eliminated when stratified by race/ethnicity (Table 1). Several SNPs and haplotypes containing these SNPs showed modest associations with spina bifida risk ( $\mathrm{p} \leq 0.05$, Table 3 and 4 ). These associations are summarized below for each gene. The results, however, are largely indicative of no or very limited associations, particularly after correction for multiple comparisons.

\section{CITED2}

Only one of the two SNPs genotyped was fully analyzed. SNP rs1131431 showed a genotypic association $(\mathrm{OR}=$ 5.32, 95\% CI: $1.04 \sim 27.30$ ) based on a recessive mode of inheritance, specifically in the White population. It is noteworthy that the confidence interval had a wide
Table 2 Demographic distribution of malformed cases and non-malformed controls, California 1990-1999

\begin{tabular}{|c|c|c|}
\hline \multirow[t]{2}{*}{ Variable } & \multicolumn{2}{|c|}{ Spina Bifida } \\
\hline & $\begin{array}{c}\text { Cases } \\
\mathrm{n}=297\left(\%^{1}\right)\end{array}$ & $\begin{array}{c}\text { Controls } \\
\mathrm{n}=300\left(\%^{1}\right)\end{array}$ \\
\hline \multicolumn{3}{|l|}{ Maternal Race/Ethnicity } \\
\hline White, non-Hispanic & $59(19.9)$ & $96(32.0)$ \\
\hline Hispanic, Native Born & $33(11.1)$ & $33(11.0)$ \\
\hline Hispanic, Foreign Born & $166(55.9)$ & $115(38.3)$ \\
\hline Black & $17(5.7)$ & $20(6.7)$ \\
\hline Asian & $9(3.0)$ & $25(8.3)$ \\
\hline Other & $9(3.0)$ & $11(3.7)$ \\
\hline \multicolumn{3}{|l|}{ Maternal age at delivery (years) } \\
\hline $13-24$ & $128(43.1)$ & $123(41.0)$ \\
\hline $25-29$ & $80(26.9)$ & $71(23.7)$ \\
\hline $30-34$ & $47(15.8)$ & $64(21.3)$ \\
\hline $35-55$ & $39(13.1)$ & $42(14.0)$ \\
\hline \multicolumn{3}{|l|}{ Maternal education } \\
\hline Less than high school & $158(53.2)$ & $110(36.7)$ \\
\hline High School & $65(21.9)$ & $81(27.0)$ \\
\hline Greater than High school & $71(23.9)$ & $109(36.3)$ \\
\hline \multicolumn{3}{|l|}{ Parity } \\
\hline 0 & $120(40.4)$ & $114(38.0)$ \\
\hline 1 & $88(29.6)$ & $101(33.7)$ \\
\hline$\geq 2$ & $86(29.0)$ & $84(28.0)$ \\
\hline \multicolumn{3}{|l|}{ Plurality } \\
\hline Singleton & $287(96.6)$ & $293(97.7)$ \\
\hline Multiple births & $8(2.7)$ & $7(2.3)$ \\
\hline \multicolumn{3}{|l|}{ Infant Sex } \\
\hline Male & $146(49.2)$ & $149(49.7)$ \\
\hline Female & $151(50.8)$ & $151(50.3)$ \\
\hline
\end{tabular}

${ }^{1}$ Percentages may not equal 100 owing to missing data or rounding.

range, suggesting that the estimate may be imprecise due to the small number of White cases and controls with the variant. This SNP, however, was previously associated with a modest increase in risk for spina bifida and congenital heart defects [14].

\section{EP300}

Among the 8 SNPs analyzed, the G allele of SNP rs4820428 was over-represented in spina bifida cases $(\mathrm{OR}=1.30,95 \% \mathrm{CI}: 1.01 \sim 1.67)$ in all subjects. Analyses showed that rs4820428 was associated with spina bifida risk under a dominant model $(\mathrm{OR}=1.5495 \% \mathrm{CI}$ : $1.10 \sim 2.17$ ). The minor allele of rs4820429 (C) showed a reduced risk in the Whites $(\mathrm{OR}=0.50,95 \% \mathrm{CI}$ : $0.26 \sim 0.50)$ and in the whole dataset $(\mathrm{OR}=0.70,95 \% \mathrm{CI}$ : $0.49 \sim 0.99)$ under a dominant model. Minor allele of rs17002284 (G) also showed a reduced risk $(\mathrm{OR}=0.43$, 95\% CI: $0.22 \sim 0.84)$, but under a recessive model. These three SNPs were in linkage disequilibrium. Subsequent analysis showed that haplotype ACGG (rs17002284rs4822006-rs4820428-rs4820429) accounted for 50.3\% of 
Table 3 Allele associations $(P<0.05)$ among subgroups

\begin{tabular}{|c|c|c|c|c|c|c|c|c|}
\hline SNP & Gene & Ethnicity & refSNP alleles & Minor Allele & $\begin{array}{l}\text { Ratio Counts } \\
\text { (Case, Control) }\end{array}$ & $P$ value & Odds Ratio & $95 \% \mathrm{Cl}$ \\
\hline rs129986 & CREBBP (CBP) & white & $\mathrm{C} / \mathrm{T}$ & $\mathrm{T}$ & 118:0, 182:10 & $0.008^{*}$ & $n / a$ & $n / a$ \\
\hline rs17616105 & CARM1 & white & $\mathrm{C} / \mathrm{T}$ & $C$ & $101: 17,136: 56$ & 0.003 & 2.45 & 1.34-4.46 \\
\hline rs303056 & TFAP2A (AP2) & white & $\mathrm{C} / \mathrm{G}$ & $C$ & $16: 102,13: 179$ & 0.046 & 2.16 & $1.00-2.15$ \\
\hline \multirow[t]{2}{*}{ rs3798691 } & TFAP2A (AP2) & Hispanic & $\mathrm{C} / \mathrm{G}$ & $C$ & $65: 333,29: 267$ & 0.013 & 1.80 & $1.13-2.87$ \\
\hline & & Hispanic-FB & $\mathrm{C} / \mathrm{G}$ & C & $52: 280,22: 208$ & 0.036 & 1.76 & $1.03-2.98$ \\
\hline rs533558 & TFAP2A (AP2) & Hispanic-NB & $\mathrm{A} / \mathrm{G}$ & G & $36: 30,24: 42$ & 0.036 & 2.10 & $1.05-4.22$ \\
\hline rs4820428 & EP300 (P300) & All subjects & $A / G$ & G & $276: 240,229: 259$ & 0.038 & 1.30 & $1.01-1.67$ \\
\hline
\end{tabular}

all haplotypes in the Whites and was over-represented in the white cases $(\mathrm{OR}=1.30,95 \% \mathrm{CI}$ : $1.01 \sim 1.67)$. It is noteworthy that this high risk haplotype contains the high risk alleles for rs17002284 (A), rs4820428 (G) as well as rs4820429 (G) (Table 5).

\section{TFAP2}

Among the 9 SNPs analyzed, rs303056 (C), rs3798691 (C), rs533558 (G) showed allelic association with spina bifida risk in Hispanics. Genotypic association analysis showed that rs3798691 was associated with spina bifida risk under a dominant model in Hispanics $(\mathrm{OR}=1.78$, 95\% CI: 1.13 2.87). SNP rs533558 also showed an association under a dominant model, but only in native-born Hispanics (OR $=3.32$, 95\%CI: 1.08 10.18). Subsequent haplotype analysis predicted two haplotype blocks in Hispanics. Haplotype AGCGC of block1 (rs537112rs533558-rs303050-rs3798694-rs3798691) had an average

Table 4 Genotype associations $(P<0.05)$ among subgroups under different modes of inheritance

\begin{tabular}{|c|c|c|c|c|c|c|}
\hline Marker & Gene & Ethnicity & $P$ value & Mode of Inheritance & $\begin{array}{c}\text { Odds Ratio } \\
{[(\text { ratio count (case, control)) }}\end{array}$ & $95 \% \mathrm{Cl}$ \\
\hline rs17002284 & EP300 & All subjects & 0.012 & Recessive & $0.43(13,29 ; 284,271)$ & $0.22-0.84$ \\
\hline \multirow[t]{3}{*}{ rs4820428 } & EP300 & All subjects & 0.011 & Additive $[(\mathrm{Dd})$ vs $(\mathrm{dd})]$ & $1.46(132,122 ; 87,117)$ & $1.00-2.11$ \\
\hline & & & & {$[(\mathrm{DD})$ vs (Dd)] } & $1.18(78,61 ; 132,122)$ & $0.78-1.79$ \\
\hline & & All subjects & 0.012 & Dominant & $1.54(210,183 ; 87,117)$ & $1.10-2.17$ \\
\hline \multirow[t]{2}{*}{ rs4820429 } & EP300 & All subjects & 0.046 & Dominant & $0.70(75,98 ; 222,202)$ & $0.49-0.99$ \\
\hline & & White & 0.041 & Dominant & $0.50(22,52 ; 37,44)$ & $0.26-0.50$ \\
\hline \multirow[t]{2}{*}{ rs1621700 } & TFAP2A & All subjects & 0.041 & Additive [(Dd) vs (dd)] & $1.30(131,118 ; 138,161)$ & $0.92-1.81$ \\
\hline & & & & {$[(\mathrm{DD})$ vs (Dd)] } & $1.33(28,19 ; 131,118)$ & $0.70-2.50$ \\
\hline \multirow[t]{4}{*}{ rs3798691 } & TFAP2A & Hispanic & 0.015 & Additive $[(\mathrm{Dd})$ vs $(\mathrm{dd})]$ & $1.78(48,23 ; 143,122)$ & $1.04-3.19$ \\
\hline & & & & {$[(\mathrm{DD})$ vs $(\mathrm{Dd})]$} & $1.40(9,3 ; 47,22)$ & $0.35-5.70$ \\
\hline & & Hispanic & 0.022 & Dominant & $1.84(56,26 ; 143,122)$ & $1.13-3.25$ \\
\hline & & Hispanic-FB & 0.049 & Dominant & $1.82(44,19 ; 122,92)$ & $1.00-3.40$ \\
\hline \multirow[t]{3}{*}{ rs533558 } & TFAP2A & Hispanic-NB & 0.038 & Additive [(Dd) vs (dd)] & $3.00(18,14 ; 6,14)$ & $0.92-9.80$ \\
\hline & & & & {$[(\mathrm{DD})$ vs (Dd)] } & $1.40(9,5 ; 18,14)$ & $0.38-5.12$ \\
\hline & & Hispanic-NB & 0.032 & Dominant & $3.32(27,19 ; 6,14)$ & $1.08-10.18$ \\
\hline \multirow[t]{4}{*}{ rs17616105 } & CARM1 & Hispanic & 0.0 & Recessive & n/a $(6,0 ; 193,148)$ & $\mathrm{n} / \mathrm{a}$ \\
\hline & & White & 0.004 & Additive [(Dd) vs (dd)] & $0.48(15,36 ; 43,50)$ & $0.23-1.00$ \\
\hline & & & & {$[(\mathrm{DD})$ vs (Dd)] } & $0.24(1,10 ; 15,36)$ & $0.03-2.04$ \\
\hline & & White & 0.010 & Dominant & $0.40(16,46 ; 43,50)$ & $0.40-0.20$ \\
\hline rs1131431 & CITED2 & White & 0.027 & Recessive & $5.32(6,2 ; 53,94)$ & $1.04-27.30$ \\
\hline \multirow[t]{6}{*}{ rs129986 } & CREBBP & All subjects & 0.005 & Additive [(Dd) vs (dd)] & $0.27(6,21 ; 291,279)$ & $0.11-0.69$ \\
\hline & & & & {$[(\mathrm{DD})$ vs $(\mathrm{Dd})]$} & $\mathrm{n} / \mathrm{a}(0,0 ; 6,21)$ & $\mathrm{n} / \mathrm{a}$ \\
\hline & & All subjects & 0.003 & Dominant & $0.27(6,21 ; 291,279)$ & $0.11-0.69$ \\
\hline & & White & 0.014 & Additive [(Dd) vs (dd)] & $\mathrm{n} / \mathrm{a}(0,10 ; 59,86)$ & $\mathrm{n} / \mathrm{a}$ \\
\hline & & & & {$[(\mathrm{DD})$ vs $(\mathrm{Dd})]$} & $\mathrm{n} / \mathrm{a}(0,0 ; 0,10)$ & $\mathrm{n} / \mathrm{a}$ \\
\hline & & White & 0.010 & Dominant & n/a $(0,10 ; 59,86)$ & $\mathrm{n} / \mathrm{a}$ \\
\hline
\end{tabular}


Table 5 Haplotype associations (only significant associations are shown)

\begin{tabular}{|c|c|c|c|c|}
\hline Haplotype Block & Frequency & P Value & Odds Ratio (case, control) & $95 \% \mathrm{Cl}$ \\
\hline \multicolumn{5}{|c|}{ TFAP2A-rs537112, rs533558, rs303050, rs3798694, and rs3798691 in Hispanic } \\
\hline AGCGC & 0.133 & 0.0205 & $1.73(63.0: 335.0,29.0: 267.0)$ & 1.08-2.77 \\
\hline GATGG & 0.516 & 0.2019 & $0.82(197.0: 201.0,161.0: 135.0)$ & $0.61-1.11$ \\
\hline GGTGG & 0.201 & 0.8135 & $0.96(78.9: 319.1,60.8: 235.2)$ & $0.66-1.39$ \\
\hline GGCAG & 0.058 & 0.4974 & $1.26(25.0: 373.0,15.0: 281.0)$ & $0.65-2.43$ \\
\hline AGCGG & 0.041 & 0.3207 & $0.69(13.9: 384.1,14.8: 281.2)$ & $0.33-1.45$ \\
\hline GGCGG & 0.028 & 0.6568 & $0.81(10.1: 387.9,9.2: 286.8)$ & $0.33-2.00$ \\
\hline AGTGG & 0.021 & 0.9639 & $0.97(8.1: 389.9,6.2: 289.8)$ & $0.34-2.79$ \\
\hline \multicolumn{5}{|c|}{ EP300- rs17002284, rs4822006, rs4820428 and rs4820429 in non-Hispanic white } \\
\hline$\overline{\text { ACGG }}$ & 0.503 & 0.0377 & $1.30(276.0: 240.0,229.0: 259.0)$ & 1.01-1.67 \\
\hline GCAG & 0.245 & 0.1665 & $0.82(117.0: 399.0,129.0: 359.0)$ & $0.61-1.09$ \\
\hline ATAC & 0.163 & 0.054 & $0.72(73.0: 443.0,91.0: 397.0)$ & $0.51-1.00$ \\
\hline ATAG & 0.072 & 0.8074 & $1.06(38.0: 478.0,34.0: 454.0)$ & $0.66-1.72$ \\
\hline ACAG & 0.015 & 0.2348 & $1.91(10.0: 506.0,5.0: 483.0)$ & $0.65-5.63$ \\
\hline
\end{tabular}

frequency of 0.133 and was associated with an increased risk, OR = $1.73(1.08-2.77)$ compared to all other haplotypes. Since no high risk SNP has been discovered, these high risk blocks will be thoroughly sequenced to search for other variants (Table 5).

\section{CREBBP}

Among the 8 SNPs analyzed, the heterozygous status of SNP rs129986 (TC) was associated with a reduced risk in all subjects $(\mathrm{OR}=0.27,95 \% \mathrm{CI}: 0.11 \sim 0.69)$. The homozygous status of the minor allele, $\mathrm{CC}$, was not observed. The TC genotype was observed in only 6 cases and they were all Hispanics.

\section{CARM1}

6 SNPs were genotyped. In Whites, the minor allele $(C)$ of rs17616105 showed a reduced risk with OR $=0.41(95 \%$ CI: 0.22 0.75). Genotype analysis showed that this effect followed a dominant inheritance model $\left(\mathrm{OR}_{\mathrm{CC}+\mathrm{CT}}=0.40\right.$, 95\% CI: $0.20 \sim 0.40$ ), and was not observed in the Hispanics.

\section{ALX1}

3 SNPs were genotyped. No association was observed between any SNP and spina bifida risk. It is known that the Cart1 knockout mouse responses to maternal supplementation with folic acid [23]. The majority of the DNA samples for this study were collected after folic acid fortification is implied in the USA, which might interact with the genotyping effect; however, our study lacks the statistic power to analyze the gene-environment interaction.

To our knowledge, this is the first study evaluating human CREBBP, EP300, CARM1, TFAP2A, and ALX1 genes as possible risk factors for spina bifida. Association between CITED2 and spina bifida has been studied before, and results from our current study are consistent with the previous findings [14]. Modest associations were observed between spina bifida risk and TFAP2A, EP300, CITED2, CREBBP1 and CARM1. No association was observed in $A L X 1$ gene. However, given the number of comparisons made, relatively few associations emerged. Although NTDs are multifactorial diseases, it cannot be excluded that the studied genes may contain rare mutations that cause some of the NTDs. With the most advanced next-generation sequencing technique being available, we may be able to discover such rare variants in our study population.

The strengths of this study include its populationbased ascertainment of cases and controls and its evaluation of race/ethnicity as potential modifiers of risk in the presence of variant genotypes and haplotypes. A major limitation of our study was the small sample size, which reduced the statistical power of analyses. Replication of some of the modest findings in a larger sample set may be warranted.

\section{Conclusions}

We have observed modest associations with risk of spina bifida in CITED2, EP300, CREBBP, TFAP2A and CARM1 genes but not $A L X 1$ gene. These modest associations were not statistically significant after correction for multiple comparisons. Future effort will focus on searching for potential functional variants and rare causal mutations in these potential candidate genes.

\section{Abbreviations}

CREB: CAMP response element binding protein; CRE: cyclic AMP-responsive; CITED2: Cbp/p300-interacting transactivator, with Glu/Asp-rich carboxyterminal domain, 2; EP300: E1A binding protein p300 (Alias: p300); CREBBP: CREB binding protein (Alias: CBP); TFAP2A: transcription factor AP-2 alpha (activating enhancer binding protein 2 alpha) (Alias: AP-2); CARM1: coactivator-associated arginine methyltransferase 1; $\boldsymbol{A L X 1}$ : ALX homeobox 1 (Alias: CART1). 


\section{Acknowledgements}

This research was supported in part by funds from NIH grants R01 NS050249 (GMS), R01 HD053509 (RMG), P20 RR017702 from the COBRE program of the National Center for Research Resources (RMG), as well as a contract from the Centers for Disease Control and Prevention, Center of Excellence Award UO1/ DD000491. We thank the California Department of Public Health Maternal Child and Adolescent Health Division for providing biological samples and data for these analyses. The findings and conclusions in this report are those of the authors and do not necessarily represent the views of the California Department of Public Health. The authors thank Ms. Consuelo Vega for her technical assistance.

\section{Author details}

'Institute of Biosciences and Technology, Texas A\&M University System Health Science Center, Houston, TX, USA. ${ }^{2}$ Stanford University, Stanford, CA, USA. ${ }^{3}$ University of Louisville Birth Defects Center, Louisville, KY, USA. ${ }^{4}$ Children's Hospital Oakland Research Institute, Oakland, CA, USA. ${ }^{5}$ Dell Pediatric Research Institute, UT Austin, Austin, TX, USA.

\section{Authors' contributions}

All authors have read and approved the final manuscript. WL performed and supervised most of the experiments, and also performed part of the statistical analysis. ARG and CJC performed most of the experiments and helped with manuscript writing. WY performed part of the statistical analysis and helped with the manuscript writing. GMS contributed to the study design and authorized the use of CBDMP samples. RMG and MMP contributed to candidate gene selection and study. RHF contributed to the study design and laboratory instruction of the molecular biology experiments. ELL reviewed and confirmed the clinical diagnosis of study subjects. $\mathrm{HZ}$ contributed to the overall study design, quality assurance of genotyping experiments, statistical analysis and most of the manuscript writing.

\section{Competing interests}

The authors declare that they have no competing interests.

Received: 1 June 2010 Accepted: 8 October 2010

Published: 8 October 2010

\section{References}

1. Bamforth SD, Braganca J, Eloranta JJ, Murdoch JN, Marques FI, Kranc KR, Farza H, Henderson DJ, Hurst HC, Bhattacharya S: Cardiac malformations, adrenal agenesis, neural crest defects and exencephaly in mice lacking Cited2, a new Tfap2 co-activator. Nat Genet 2001, 29:469-474.

2. Barbera JP, Rodriguez TA, Greene ND, Weninger WJ, Simeone A, Copp AJ, Beddington RS, Dunwoodie S: Folic acid prevents exencephaly in Cited2 deficient mice. Hum Mol Genet 2002, 11:283-293.

3. Bhattacherjee V, Horn KH, Singh S, Webb CL, Pisano MM, Greene RM: CBP/ p300 and associated transcriptional co-activators exhibit distinct expression patterns during murine craniofacial and neural tube development. Int J Dev Biol 2009, 53:1097-1104.

4. Petrij F, Giles RH, Dauwerse HG, Saris JJ, Hennekam RC, Masuno M, Tommerup N, van Ommen GJ, Goodman RH, Peters DJ, et al: RubinsteinTaybi syndrome caused by mutations in the transcriptional co-activator CBP. Nature 1995, 376:348-351

5. Tanaka Y, Naruse I, Hongo T, Xu M, Nakahata T, Maekawa T, Ishii S: Extensive brain hemorrhage and embryonic lethality in a mouse null mutant of CREB-binding protein. Mech Dev 2000, 95:133-145.

6. Weninger WJ, Lopes Floro K, Bennett MB, Withington SL, Preis JI, Barbera JP, Mohun TJ, Dunwoodie SL: Cited2 is required both for heart morphogenesis and establishment of the left-right axis in mouse development. Development 2005, 132:1337-1348.

7. Yadav N, Lee J, Kim J, Shen J, Hu MC, Aldaz CM, Bedford MT: Specific protein methylation defects and gene expression perturbations in coactivator-associated arginine methyltransferase 1-deficient mice. Proc Natl Acad Sci USA 2003, 100:6464-6468.

8. Yao TP, Oh SP, Fuchs M, Zhou ND, Ch'ng LE, Newsome D, Bronson RT, Li E, Livingston DM, Eckner R: Gene dosage-dependent embryonic development and proliferation defects in mice lacking the transcriptional integrator p300. Cell 1998, 93:361-372.
9. Zhao Q, Behringer RR, de Crombrugghe B: Prenatal folic acid treatment suppresses acrania and meroanencephaly in mice mutant for the Cart1 homeobox gene. Nat Genet 1996, 13:275-283.

10. Kitabayashi I, Aikawa Y, Nguyen LA, Yokoyama A, Ohki M: Activation of AML1-mediated transcription by MOZ and inhibition by the MOZ-CBP fusion protein. EMBO J 2001, 20:7184-7196.

11. Xu W, Chen H, Du K, Asahara H, Tini M, Emerson BM, Montminy M, Evans RM: A transcriptional switch mediated by cofactor methylation. Science 2001, 294:2507-2511.

12. Jayne $\mathrm{S}$, Rothgiesser KM, Hottiger MO: CARM1 but not its enzymatic activity is required for transcriptional coactivation of NF-kappaBdependent gene expression. J Mol Biol 2009, 394:485-495.

13. Chen SL, Loffler KA, Chen D, Stallcup MR, Muscat GE: The coactivatorassociated arginine methyltransferase is necessary for muscle differentiation: CARM1 coactivates myocyte enhancer factor-2. J Biol Chem 2002, 277:4324-4333.

14. Volcik KA, Zhu H, Finnell RH, Shaw GM, Canfield M, Lammer EJ: Evaluation of the Cited 2 gene and risk for spina bifida and congenital heart defects. Am J Med Genet A 2004, 126A:324-325.

15. Yin Z, Haynie J, Yang X, Han B, Kiatchoosakun S, Restivo J, Yuan S, Prabhakar NR, Herrup K, Conlon RA, et al: The essential role of Cited2, a negative regulator for HIF-1alpha, in heart development and neurulation. Proc Natl Acad Sci USA 2002, 99:10488-10493.

16. Braganca J, Eloranta JJ, Bamforth SD, Ibbitt JC, Hurst HC, Bhattacharya S: Physical and functional interactions among AP-2 transcription factors, p300/CREB-binding protein, and CITED2. J Biol Chem 2003, 278:16021-16029.

17. Zhang J, Hagopian-Donaldson S, Serbedzija G, Elsemore J, PlehnDujowich D, McMahon AP, Flavell RA, Williams T: Neural tube, skeletal and body wall defects in mice lacking transcription factor AP-2. Nature 1996, 381:238-241.

18. Schorle $H$, Meier $P, B$ Buchert $M$, Jaenisch $R$, Mitchell PJ: Transcription factor AP-2 essential for cranial closure and craniofacial development. Nature 1996, 381:235-238.

19. Nottoli T, Hagopian-Donaldson S, Zhang J, Perkins A, Williams T: AP-2-null cells disrupt morphogenesis of the eye, face, and limbs in chimeric mice. Proc Natl Acad Sci USA 1998, 95:13714-13719.

20. lioka T, Furukawa K, Yamaguchi A, Shindo H, Yamashita S, Tsukazaki T: P300/ CBP acts as a coactivator to cartilage homeoprotein-1 (Cart1), paired-like homeoprotein, through acetylation of the conserved lysine residue adjacent to the homeodomain. J Bone Miner Res 2003, 18:1419-1429.

21. Zhao GQ, Zhou X, Eberspaecher H, Solursh M, de Crombrugghe B: Cartilage homeoprotein 1, a homeoprotein selectively expressed in chondrocytes. Proc Natl Acad Sci USA 1993, 90:8633-8637.

22. Zhao GQ, Eberspaecher $H$, Seldin MF, de Crombrugghe $B$ : The gene for the homeodomain-containing protein Cart-1 is expressed in cells that have a chondrogenic potential during embryonic development. Mech Dev 1994, 48:245-254.

23. Harris MJ: Insights into prevention of human neural tube defects by folic acid arising from consideration of mouse mutants. Birth Defects Res A Clin Mol Teratol 2009, 85:331-339.

24. Croen LA, Shaw GM, Jensvold NG, Harris JA: Birth defects monitoring in California: a resource for epidemiological research. Paediatr Perinat Epidemiol 1991, 5:423-427.

25. De La Vega FM, Isaac HI, Scafe CR: A tool for selecting SNPs for association studies based on observed linkage disequilibrium patterns. Pac Symp Biocomput 2006, 487-498.

26. Baluja KF, Park J, Myers D: Inclusion of immigrant status in smoking prevalence statistics. Am J Public Health 2003, 93:642-646.

27. Curry CJ, Lammer EJ, Nelson V, Shaw GM: Schizencephaly: heterogeneous etiologies in a population of 4 million California births. Am J Med Genet A 2005, 137:181-189.

28. Benjamini $Y$, Hochberg $Y$ : Controlling the false discovery rate: a practical and powerful approach to multiple testing. Journal of the Royal Statistical Society Series B, Statistical methodology 1995, 57:289.

\section{Pre-publication history}

The pre-publication history for this paper can be accessed here: http://www.biomedcentral.com/1471-2350/11/141/prepub

\section{doi:10.1186/1471-2350-11-141}

Cite this article as: Lu et al:: Genes encoding critical transcriptional activators for murine neural tube development and human spina bifida: a case-control study. BMC Medical Genetics 2010 11:141. 